\title{
Kneading sequences for double standard maps
}

\author{
by \\ Michael Benedicks (Stockholm) and \\ Ana Rodrigues (Indianapolis, IN, and Porto) \\ Dedicated to our common friend, Michat Misiurewicz, on the occasion \\ of his 60th birthday with an appreciation of all what he has done \\ for us and our field
}

\footnotetext{
Abstract. We investigate the symbolic dynamics for the double standard maps of the circle onto itself, given by $f_{a, b}(x)=2 x+a+(b / \pi) \sin (2 \pi x)(\bmod 1)$, where $b=1$ and $a$ is a real parameter, $0 \leq a<1$.
}

1. Introduction. In the family of double standard maps of the circle onto itself, given by

$$
f_{a, b}(x)=2 x+a+(b / \pi) \sin (2 \pi x)(\bmod 1),
$$

where the parameters $a, b$ are real and $0 \leq b \leq 1$, tongues (sets of parameter values for which there is an attracting periodic point) appear (see Misiurewicz-Rodrigues [17, 18, 19]).

The aim of this paper is to study the symbolic dynamics for the double standard family assuming $b=1$. We define $f_{a}=f_{a, 1}$ for $f_{a, b}$ in (1.1). Moreover, we relate this to the "Real Fatou Conjecture", that is, the density of parameters with attractive periodic orbits.

In the classical theory of Milnor-Thurston the symbolic coding is associated to the two intervals where the restriction of the map to each of them is increasing or decreasing (the basic background for symbolic dynamics and kneading theory may be found in [7]). Consider the double standard family (1.1) with $b=1$ and $a \in[0,1)$. Then for each value of the parameter $a$ the map is increasing for all values of $x \in[0,1]$ except for the values of the parameter for which there is an attractive periodic orbit of period 1 (see [18]).

2010 Mathematics Subject Classification: Primary 37E10.

Key words and phrases: circle maps, covering maps, double standard maps, symbolic dynamics, kneading theory. 
The explicit computation of the boundary of the period 1 tongue (see [19]) gives:

$$
a=\frac{1}{2} \pm \frac{\sqrt{4 b^{2}-1}-\arctan \sqrt{4 b^{2}-1}}{2 \pi},
$$

which allows us to compute the interval $\left[a_{0}^{\prime}, a_{0}\right]$ for which we have an attractive periodic orbit of period one. We get $a_{0} \simeq 0.65$, the bifurcation point for the period 1 tongue for $a>1 / 2$, and $a_{0}^{\prime}=1-a_{0}$.

For $a_{0}<a<1, f_{a}$ has a unique fixed point, which we will denote by $p(a)$. This follows from the bifurcation behavior of the fixed point(s). For $a=1 / 2, f_{a}$ has three fixed points, one at $x=1 / 2$ and two repelling fixed points. According to the Implicit Function Theorem this behavior persists for $a_{0}^{\prime}<a<a_{0}$. For $a=a_{0}$, the two rightmost fixed points go through a saddle node bifurcation and disappear. Since the only bifurcation of fixed points for $1 / 2<a<1$ appears at $a=a_{0}$ it is clear that there is at most one fixed point in this interval, the continuation of the left fixed point that exists for $1 / 2<a<a_{0}$. We denote this fixed point by $p(a)$. We will also prove that $p(a)$ for $a_{0}<a<1$ has a unique preimage different from $p(a)$. We denote this preimage by $q(a)$. For $0<a<a_{0}^{\prime}$ the situation is completely symmetric.

We use a symbolic coding related to Yoccoz partitions of the interval [23], but in our case we will apply it to the circle. Let $J_{0}=(p(a), q(a))$ for $a>1 / 2$, where the circle segments are chosen so that they have positive orientation on the circle, and let $J_{1}=\operatorname{int}\left(\mathbb{T} \backslash J_{0}\right)$. In the case $0<a<a_{0}^{\prime}$, $J_{0}=[0, q(a)) \cup(p(a), 1)$ where the circle is represented by the half-open fundamental domain $[0,1)$ and as before $J_{1}$ is the interior of its complement.

A more geometric way to express the same is to say that $J_{0}$ is the positively oriented (counterclockwise) open arc on the unit circle from $p(a)$ to $q(a)$, and $J_{1}$ is the interior of its complement.

For a given initial point $x$ on the circle such that its orbit does not land on $p$ or $q$ let

$$
i_{n}(x)= \begin{cases}0 & \text { if } f_{a}^{n}(x) \in J_{0} \\ 1 & \text { if } f_{a}^{n}(x) \in J_{1} .\end{cases}
$$

For a point that eventually hits $p$ after possibly passing through $q$, the coding is so far not defined. For these orbits, we define the coding by either $i_{0} \ldots i_{n} 0 \overline{1}$ or $i_{0} \ldots i_{n} 1 \overline{0}$, and we identify these sequences. Note that this is exactly the same identification as is made of the binary expansions

$$
0 . i_{0} \ldots i_{n} 0 \overline{1} \text { and } 0 . i_{0} \ldots i_{n} 1 \overline{0}
$$

when they are interpreted as real numbers.

Thus, we associate with each $x \in \mathbb{T}$ a finite or infinite sequence of the symbols 0,1 called its itinerary. We denote by $I(x)$ the sequence $\left\{i_{n}(x)\right\}_{n=0}^{\infty}$ and this sequence is also naturally identified with a real number in $[0,1]$. 
As usual the kneading sequence will be the itinerary of the critical value $f_{a}(1 / 2)=a$ and we denote it by $K\left(f_{a}\right)$. We will sometimes also use the notation $K(a)=K\left(f_{a}\right)$, in particular when we consider the function $a \mapsto$ $K(a)$.

The paper is organized as follows. In Section 2 we introduce the symbolic coding and we study the monotonicity of the itinerary map and of the kneading sequence. In Section 3 we investigate some properties of the periodic kneading sequences. In Section 4 we investigate some properties of the aperiodic kneading sequences and we show how to connect our work to the "Real Fatou Conjecture", which in the present setting is a result by Levin and van Strien [11, Theorem C]. In Section 5 we state some results concerning the abundance of positive Lyapunov exponents and invariant measures. Finally, in Section 6 we state some conjectures.

2. Kneading sequences. Let $I(x), I(y)$ be two sequences of the symbols $\{0,1\}$ such that $I(x) \neq I(y)$. Suppose

$$
I(x)=i_{0}^{1} i_{1}^{1} i_{2}^{1} \cdots i_{n}^{1}, \quad I(y)=i_{0}^{2} i_{1}^{2} i_{2}^{2} \cdots i_{n}^{2},
$$

where $i_{n}$ is the smallest index for which $i_{n}^{1} \neq i_{n}^{2}$. We order the sequences (2.1) lexicographically: if $i_{n}^{1}=0, i_{n}^{2}=1$ then $I(x)<I(y)$. It is obvious that this order coincides with the order of the real numbers corresponding to the symbol sequences interpreted as binary expansions. It is clear that the map $x \mapsto I(x)$ is continuous, where the topology on the kneading sequences is the topology of real numbers.

We prove the following lemma:

LemMa 2.1. For $f=f_{a, b}$ as in (1.1) with $b=1$ and for a fixed value of the parameter $a$, if $x<y$ then $I(x) \leq I(y)$.

Proof. By the continuity of $x \mapsto I(x)$ it is enough to treat the case when $x$ and $y$ are not binary rationals.

We will prove

$$
I(x)>I(y) \Rightarrow x>y .
$$

We argue by induction on the index where $I(x)$ and $I(y)$ differ. For $n=0$, (2.2) is obvious. Assume it has been proved for $n-1$ and we wish to prove it for $n$. But $I(x)>I(y) \Rightarrow I(f(x))>I(f(y))$. By (2.2) for $n-1$, we conclude that $f(x)>f(y)$. But by the strict monotonicity of $f$ it now follows that $x>y$.

We also define an order on the kneading sequences in exactly the same way as we defined an order on the itineraries.

We have the following lemma: 
Lemma 2.2. Fix $b=1$ in (1.1). Assume that $1 / 2<a_{*}<1$ does not belong to the period 1 tongue and that $f_{a_{*}}$ has the property that the critical point $1 / 2$ is eventually fixed, i.e. there is an integer $j$ so that $f_{a_{*}}^{j}(1 / 2)=p$. Then $a \mapsto K\left(f_{a}\right)$ is strictly monotonic at $a_{*}$. More precisely, there is a real number $\delta>0$ so that

$$
a_{*}-\delta<a_{1}<a_{*}<a_{2}<a_{*}+\delta<1 \Rightarrow K\left(f_{a_{1}}\right)<K\left(f_{a_{2}}\right) .
$$

Proof. Outside the period 1 tongue we have $a_{0}<a<1$. We begin by proving that for $a$ in this interval we have $0<p(a)<1 / 6$ and that $p(a)$ decreases as a function of $a$. Consider the lift $F_{a}$ of (1.1) to the real line. The fixed point of $f_{a}$ satisfies

$$
F_{a}(x)=2 x+a+\frac{1}{\pi} \sin (2 \pi x)=x+1 .
$$

Consider the function $G_{a}(x)=F_{a}(x)-x-1$ and $1 / 2<a<1$. We have $G_{a}(0)=a-1<0$ and $G_{a}(1 / 6)=-5 / 6+a+\sqrt{3} / 2 \pi>0$. Thus, there is $0<c<1 / 6$ such that $G_{a}(c)=F_{a}(c)-c-1=0$.

Differentiating $F_{a}(p(a))=p(a)+1$ with respect to $a$ yields

$$
[1+2 \cos (2 \pi p(a))] \frac{d p(a)}{d a}+1=0 .
$$

Now if $0 \leq p(a)<1 / 6$ then $\cos (2 \pi p(a))>-1 / 2$ and we conclude that $d p(a) / d a<0$. Since $p(a)=0$ only for $a=1$, we conclude that $0<p(a)$ $<1 / 6$.

We now prove that $q(a)$ exists and decreases as a function of $a$. For $a_{0}<a<a_{0}+\epsilon$ with $\epsilon$ small we have $q(a) \simeq 0.61$. For $1-\epsilon<a<1, q(a)$ is small and positive. Since $q(a)$ satisfies

$$
F_{a}(q(a))=p(a)+1,
$$

differentiating with respect to $a$ and inserting the expression for $d p(a) / d a$ yields

$$
\left[(2+2 \cos (2 \pi q(a))] \frac{d q(a)}{d a}=-1-\frac{d p(a)}{d a}=-\frac{2 \cos (2 \pi p(a))}{1+2 \cos (2 \pi p(a))} .\right.
$$

From the Implicit Function Theorem it follows that $q(a)$ exists in the relevant interval $a_{0}<a<1$. Using the fact that $0<p(a)<1 / 6$ for $a_{0}<a<1$ and the behavior at the end points we conclude that $d q(a) / d a<0$ for $a_{0}<a<1$.

We want to prove that $f_{a}^{n}(1 / 2)$ increases as a function of $a$. Recall the proof of Lemma 2.6 from [18]: since $f_{a}^{n}(x)=f_{a}^{n-1}\left(f_{a}(x)\right)$, we have

$$
\frac{\partial f_{a}^{n}}{\partial a}(x)=\frac{\partial f_{a}^{n-1}}{\partial a}\left(f_{a}(x)\right)+\left(f_{a}^{n-1}\right)^{\prime}\left(f_{a}(x)\right) \frac{\partial f_{a}}{\partial a}(x) .
$$


By (1.1), $\frac{\partial f_{a}}{\partial a}(y)=1$ for every $y$, so by induction we obtain

$$
\frac{\partial f_{a}^{n}}{\partial a}(x)=\sum_{k=0}^{n-1}\left(f_{a}^{k}\right)^{\prime}\left(f_{a}^{n-k}(x)\right) .
$$

Since $f_{a}^{\prime}$ is non-negative everywhere, so is $\left(f_{a}^{k}\right)^{\prime}$. Moreover, if $k=0$ then $\left(f_{a}^{k}\right)^{\prime} \equiv 1$.

Set $\xi_{n}(a)=f_{a}^{n}(1 / 2)$. We assume that $a_{*}$ is such that we do not have a period 1 tongue. Let $a$ be very close to $a_{*}$. When $a$ increases, $\xi_{n}(a)=f_{a}^{n}(1 / 2)$ hits $p$ for $a=a_{*}$ and there is a change in the $n$th symbol of the kneading sequence. But actually $\xi_{n-1}$ must simultaneously hit the preimage $q$. This means that there are two symbols that change simultaneously. The monotonicity properties of $\xi_{n}(a), p(a)$ and $q(a)$ proved above show that $i_{n}$ changes from 1 to 0 but $i_{n-1}$ changes from 0 to 1 . The latter change of $i_{n-1}$ has more weight since it is of lower index. Hence the kneading must increase at $a_{*}$ when $a$ increases.

As a consequence we obtain the following statement on the monotonicity of kneading sequences for the family $f_{a}$.

THEOREM 2.3. Let $f_{a_{1}}, f_{a_{2}}$ belong to the double standard family. Then

(i) $K\left(f_{a_{1}}\right)<K\left(f_{a_{2}}\right)$ implies $a_{1}<a_{2}$;

(ii) $a_{1}<a_{2}$ implies $K\left(f_{a_{1}}\right) \leq K\left(f_{a_{2}}\right)$.

Proof. We first prove the theorem in the case $a_{0}<a_{1}, a_{2}$. The case $a_{1}, a_{2}<a_{0}^{\prime}$ will be postponed until after the proof of Lemma 2.4. Let $n$ be the first index where $K\left(f_{a_{1}}\right)=\left(k_{i}^{1}\right)_{i=0}^{\infty}$ and $K\left(f_{a_{2}}\right)=\left(k_{i}^{2}\right)_{i=0}^{\infty}$ differ. The proof of (i) goes by induction on $n$. To begin, the statement is true for $n=1$ by direct computation. Suppose that (i) is proven for all indices of change $\leq n-1$ and let $n \geq 2$.

Suppose that $a_{1}<a_{2}$ and $K\left(a_{1}\right)>K\left(a_{2}\right)$ and suppose that $k_{1}^{1} k_{2}^{1} \ldots k_{n}^{1}$ and $k_{1}^{2} k_{2}^{2} \ldots k_{n}^{2}$ differ in the $n$th binary digit. This digit changes at points $\left\{a_{i}\right\}$, where the critical point is preperiodic. By the analyticity there are only finitely many of these points. Note that at each of these points, the map is strictly locally increasing by Lemma 2.3. If there are more than one point $\left\{a_{i}^{\prime}\right\}$ this would mean that more than one of the digits $\left\{k_{i}^{1}\right\}_{i=1}^{n-1}$ change in a decreasing order as a function of $a$, which contradicts the induction assumption. If there is only one point $a_{1}^{\prime}$ the local strict increase at that point contradicts $K\left(a_{1}\right)>K\left(a_{2}\right)$.

Now the proof of (ii) is immediate. We again argue by contradiction. If we assume $K\left(f_{a_{1}}\right)>K\left(f_{a_{2}}\right)$, by (i) we get $a_{1}>a_{2}$, which contradicts the assumption $a_{1}<a_{2}$.

LEMmA 2.4. Assume that $1 / 2<a_{1}<1, a_{2}=1-a_{1}$ and $K\left(f_{a_{1}}\right), K\left(f_{a_{2}}\right)$ are the corresponding kneading sequences interpreted as real numbers. Then $K\left(f_{a_{1}}\right)=1-K\left(f_{a_{2}}\right)$. 
Proof. Assume that $1 / 2<a_{1}<1$ and $a_{2}=1-a_{1}$. We start by proving that if $p\left(a_{1}\right), p\left(a_{2}\right)$ are fixed points of $f_{a_{1}}, f_{a_{2}}$, respectively, then $p\left(a_{2}\right)=$ $1-p\left(a_{1}\right)$ and $q\left(a_{2}\right)=1-q\left(a_{1}\right)$. Consider the lifting $F_{a}$ of $f_{a}$ to the real line. A fixed point of $f_{a}$ satisfies

$$
F_{a}=2 x+a+\frac{1}{\pi} \sin (2 \pi x)=x+1 .
$$

Consider the map $G(a, x)=F_{a}(x)-x-1$. If $\left(a_{1}, p\left(a_{1}\right)\right)$ is a solution of $G(a, x)=0$ then $\left(1-a_{1}, 1-p\left(a_{1}\right)\right)$ is also a solution. Assume $G_{a_{1}}\left(p\left(a_{1}\right)\right)=$ $p\left(a_{1}\right)+a_{1}+\pi^{-1} \sin \left(2 \pi p\left(a_{1}\right)\right)-1=0$. Then $G_{1-a_{1}}\left(1-p\left(a_{1}\right)\right)=-G_{a_{1}}\left(p\left(a_{1}\right)\right)=0$.

We now prove that if $p\left(a_{1}\right)<f_{a_{1}}^{n}(1 / 2)<q\left(a_{1}\right)$, then $q\left(a_{2}\right)<f_{a_{2}}^{n}(1 / 2)<$ $p\left(a_{2}\right)$, for $a_{2}=1-a_{1}$. We see that

implies

$$
p\left(a_{1}\right)<f_{a_{1}}^{n}(1 / 2)<q\left(a_{1}\right)
$$

and so

$$
1-p\left(a_{2}\right)<f_{1-a_{2}}^{n}(1 / 2)<1-q\left(a_{2}\right)
$$

We will prove that

$$
q\left(a_{2}\right)<1-f_{1-a_{2}}^{n}(1 / 2)<p\left(a_{2}\right) .
$$

by induction on $n$. For $n=1$ we have $f_{a_{2}}^{n}(1 / 2)=1-f_{1-a_{2}}^{n}(1 / 2)=a_{2}$ $(\bmod 1)$. Assume $(2.4)$ holds for $n$. We show it holds for $n+1$ :

$$
\begin{aligned}
f_{a_{2}}\left(f_{a_{2}}^{n}(1 / 2)\right) & =f_{a_{2}}\left(1-f_{1-a_{2}}^{n}(1 / 2)\right)=f_{a_{2}}\left(-f_{a_{2}}^{n}(1 / 2)\right)=-f_{-a_{2}}\left(f_{1-a_{2}}^{n}(1 / 2)\right) \\
& =-f_{1-a_{2}}\left(f_{1-a_{2}}^{n}(1 / 2)\right)=-f_{1-a_{2}}^{n+1}(1 / 2)=1-f_{1-a_{2}}^{n+1}(1 / 2) .
\end{aligned}
$$

Hence we see that for the $n$th item in the kneading sequences if $k_{n}\left(f_{a_{1}}\right)=0$ then $k_{n}\left(f_{a_{2}}\right)=1$ and vice-versa, so $K\left(f_{a_{1}}\right)=1-K\left(f_{a_{2}}\right)$.

Proof of Theorem 2.3 for $a<1 / 2$. We suppose that $0<a_{1}<a_{2}<a_{0}^{\prime}$. Then $1-a_{2}<1-a_{1}$ and by Theorem 2.3 for $a>1 / 2$ it follows that $K\left(1-a_{2}\right) \leq K\left(1-a_{1}\right)$ and (ii) follows. The proof of (i) is similar.

The next lemma will be quite useful. Since $K(a)$ for $0 \leq a \leq a_{0}^{\prime}$ and $a_{0} \leq a<1$, may be interpreted as real numbers, it will immediately imply an intermediate value theorem for kneading sequences:

Lemma 2.5. The map $a \mapsto K(a)$ is continuous, where the topology on the space of kneading sequences is given by the topology of the real numbers in the interval $[0,1]$.

Proof. The argument is standard; see e.g. the proof of [7, Proposition III.1.2]. One sees that $A_{0}=\left\{a \mid K(a)>K_{0}\right\}$ and $A_{0}^{\prime}=\left\{a \mid K(a)<K_{0}\right\}$ are both open by verifying that each point in these sets is contained in an open set of the type $\left\{a \mid k_{j}(a)=k_{j}^{0}, j=0, \ldots, n\right\}$, which in turn is contained in the corresponding set $A_{0}$ and $A_{0}^{\prime}$ respectively. 
3. Periodic kneading sequences. In this section we prove that the kneading sequence corresponding to a periodic orbit is a periodic sequence. Furthermore, we prove that it corresponds to the binary expansion of the rational number assigning the order of the given tongue as described in [19].

Let $W$ be an open subset of $\mathbb{T}$. We say that $\left.f\right|_{W}$ has a sink if there is an open interval $K \subset W$ such that $f(K) \subset K$ for some $n \geq 1$ and $f^{j}(K) \subset W$, $j=1, \ldots, n-1$ (and hence for all $j$ ).

We note the following lemma:

LEMma 3.1. If $f=f_{a}$ is a map from the double standard family and $W$ is an open subset of $\mathbb{T}$ such that $\left.f\right|_{W}$ has a sink then $\bar{W}$ contains an attractive periodic orbit.

Proof. If $f$ has a sink then $g=\left.f^{n}\right|_{K}$ is a homeomorphism of $K$ into itself. We want to show that $g$ has a stable fixed point in $\bar{K}$. It is obvious that there is a point $x \in \bar{K}$ such that $g(x)=x$. We pick the leftmost fixed point of this type. If $g$ maps $\bar{K}$ into $K$ this fixed point lies in $K$ and must satisfy $0 \leq g^{\prime}(x) \leq 1$. If $0 \leq g^{\prime}(x)<1$, we are done. If $g^{\prime}(x)=1$ then $x$ is stable from the left.

If $g(x)=x$ for some $x \in \partial \bar{K}$ then we argue as follows. Suppose $x$ is the left endpoint. If $g^{\prime}(x) \leq 1$ and $g(y)<y$ for $y$ near $x$ in $K$ then $x$ is one-sided stable. If $g(y)>y$ for $y$ near $x$ or $g^{\prime}(x)>1$ then we argue as in the case $g(\bar{K}) \subset K$. The right endpoint is handled in a symmetric fashion.

We next turn to a result which in the unimodal case is due to Guckenheimer [9].

THEOREM 3.2. If $f_{a}$ belongs to the double standard family then:

(i) $f_{a}$ has a stable periodic orbit if and only if $K\left(f_{a}\right)$ is periodic.

(ii) If $K\left(f_{a_{1}}\right)$ is periodic and $K\left(f_{a_{1}}\right)=K\left(f_{a_{2}}\right)$ and the periodic orbits of $f_{a_{1}}$ and $f_{a_{2}}$ are two-sided attracting, then $f_{a_{1}}$ and $f_{a_{2}}$ are topologically conjugate.

REMARK. Theorem 3.2(ii) implies that the $f_{a}$ 's with parameters in the interior of the tongues are topologically conjugate. The functions with parameters on the endpoints of the tongues have one-sided attracting periodic orbits and are not conjugate to those with parameters in the interior.

Proof of Theorem 3.2. We start by proving (i). Assume that $f$ has a stable periodic orbit of period $n$. Let $x$ be a point on the orbit. Let $y$ be a critical point of $f^{n}$ so that $0<\left|D f^{n}\right| \leq 1$ on $(x ; y)((x ; y)$ is $(x, y)$ or $(y, x)$, depending on whether $x<y$ or $x>y)$. Such a critical point exists since there is always a critical point in the immediate basin of an attractive periodic orbit by Singer's theorem. 
Let $j$ be such that $f^{j}(y)=1 / 2$. Now $f^{r n}(y)$ converges monotonously to $x$ when $r \rightarrow \infty$. Therefore $f^{j}\left(f^{r n}(y)\right)=f^{r n}(1 / 2)$ converges monotonously to $f^{j}(x)$.

Assume now $p \in\left(f^{k}(1 / 2) ; f^{k+j}(x)\right)$ for some $k$. This implies $p \in\left(f^{k+j}(y)\right.$; $\left.f^{k+j}(x)\right)$; in particular when $r n>k+j$ there is a $z$ in $(y ; x)$ such that $f^{r n}(z)=p$. But this contradicts the fact that the entire interval $(y ; x)$ is attracted to the attractive periodic orbit. Hence the case $p \in\left(f^{k}(1 / 2) ; f^{k+j}(x)\right)$ does not occur. We conclude that $f(1 / 2)$ and $f^{j+1}(x)$ have the same itinerary. Hence $I(f(1 / 2))$ is periodic (and not only eventually periodic).

If on the other hand $I(f(1 / 2))$ is periodic then all points in the interval $\left(f^{m+1}(1 / 2) ; f(1 / 2)\right)$ have the same itineraries. Either $f^{m+1}(1 / 2)=f(1 / 2)$ and we have a superattractive orbit. If not, let us for convenience assume that $f^{m+1}(1 / 2)<f(1 / 2)$. Then the interval $\left(f^{m+1}(1 / 2), f(1 / 2)\right)$ is mapped to $\left(f^{2 m+1}(1 / 2), f^{m+1}(1 / 2)\right)$. In the same way

$$
\left(f^{j m+1}(1 / 2), f^{(j-1) m+1}(1 / 2)\right) \mapsto\left(f^{(j+1) m+1}(1 / 2), f^{j m+1}(1 / 2)\right) .
$$

It follows that the sequence $f^{j m+1}(1 / 2)$ is monotonous as a function of $j$ and it converges to a point. This must be a point of an attractive periodic orbit, which then obviously exists. The case $f^{m+1}(1 / 2)>f(1 / 2)$ is completely analogous.

Now we turn to the proof of (ii). We follow the proof of Theorem II.6.3 in [7], but the present case is actually easier since $D f(x)>0$. Let us write $f=f_{a_{1}}$ and $g=f_{a_{2}}$. Let $U_{f}$ be the stable neighborhood of $1 / 2$ for $f$. Then $f^{n}(x) \in U_{f}$ if and only if $\mathcal{S}^{n+1}(I(x))=I_{f}(f(1 / 2))=K(f)$. We set

$$
E_{f}=\left\{x: f^{n}(x) \text { does not tend to the stable periodic orbit }\right\} .
$$

Hence we can determine whether $x \in E_{f}$ from its itinerary. Note that $x \mapsto$ $I_{f}(x)$ is injective for $x \in E_{f}$. This follows since for all $x \in E_{f}$ the orbit of $x$ must avoid the attractive neighborhood of $1 / 2$. Hence Mañé's theorem (see e.g. [15, Theorem III.5.1]) is applicable. We conclude that there exist constants $C>0$ and $\lambda>1$ so that

$$
\left|D f^{n}(x)\right| \geq C \lambda^{n} \quad \forall n \geq 0 \forall x \in E_{f},
$$

and this forbids the existence of an interval of points with constant itinerary. The same considerations apply to $g$ and hence we can define a homeomorphism $h: E_{f} \rightarrow E_{g}$ by the property $I_{f}(x)=I_{g}(h(x))$. We extend $h$ to the set $\bigcup_{i>0} f^{-i}\left(U_{f}\right)$ assuming that we have already constructed a topological equivalence $h$ from $\left.f^{n}\right|_{U_{f}}$ to $\left.g^{n}\right|_{U_{g}}$, where $n$ is the smallest integer for which $f^{n}\left(U_{f}\right) \subset U_{f}$ (and hence also $g^{n}\left(U_{g}\right) \subset U_{g}$ ). For each component $K$ of $f^{-i}\left(U_{f}\right)$ other than $U_{f}$ there is a $j \leq i$ with $f^{j}$ mapping $K$ homeomorphically into $U_{f}$. Then we define $h$ for $x \in K$ by $h\left(f^{j}(x)\right)=g^{j}(h(x))$ and the requirement that the itineraries $I_{f}(x)$ and $I_{g}(h(x))$ are the same (this 
identifies the component). It is easy to see that $h$ defined this way is a topological equivalence. It remains to define $h$ on $U_{f}$. The set $U_{f}$ must always be of the form $(p, q)$, where $f^{n}(p)=p$ and $f^{n}(q)=q$ with an attractive periodic point in the interior of $U_{f}$ and $U_{g}$ is of the same form. The usual fundamental domains argument in the proof of Hartman's theorem is then used to define the conjugacy $h$ on $U_{f}$.

The order of the tongues for the family (1.1) corresponds to the order of rational numbers with denominator $2^{n}-1$. Let $F_{a, b}$ be the lift of $f_{a, b}$ to the real line. The limit

$$
\Phi_{a, b}(x)=\lim _{j \rightarrow \infty} \frac{F_{a, b}^{j}(x)}{2^{j}},
$$

where each $F_{a, b}$ is continuous increasing (as a function of $x$ ) and $F_{a, b}(x+k)=$ $F_{a, b}(x)+2 k$ for every integer $k$, exists uniformly in $x$ (see [19, Lemma 3.1]).

The fact that the periodic part of the binary expansion of $\Phi_{a}(x)$ gives us a periodic coding in 0's and 1's suggests that there is a relation between a periodic kneading and the periodic part of this binary expansion.

THEOREM 3.3. If a is such that $f_{a}$ has a periodic orbit of period $n(n>1)$ then $K\left(f_{a}\right)$ corresponds to the shift of the binary expansion of $\Phi_{a}(1 / 2)=$ $k /\left(2^{n}-1\right)$ where $k$ is a natural number and $k=1, \ldots, 2^{n}-2$.

Proof. We make a linear conjugacy (or rotation)

$$
T(\xi)=\xi+p .
$$

The map in the new coordinates is

$$
\hat{f}(\xi)=T^{-1} \circ f \circ T,
$$

and it has a fixed point at $\xi=0$.

We want to compute the limit

$$
\lim _{j \rightarrow \infty} \frac{F_{a}^{j}(x)}{2^{j}},
$$

in particular for $x=1 / 2$ we want to show that the itinerary goes with this limit.

The point $q$ is defined by $F(q)=1$. We have

$$
F(x)= \begin{cases}f(x), & 0 \leq x \leq q, \\ f(x)+1, & q \leq x \leq 1,\end{cases}
$$

and

$$
F(x)= \begin{cases}f(x-k)+k, & k \leq x \leq q+k \\ f(x-k)+k+1, & q+k \leq x \leq k+1 .\end{cases}
$$


For an itinerary $\left\{i_{j}\right\}_{j=0}^{\infty}$ we have the recursion formula

$$
\left\{\begin{array}{l}
a_{j+1}=2 a_{j}+i_{j} \\
\xi_{j+1}=f\left(\xi_{j}\right)
\end{array}\right.
$$

for $j=0,1,2$, which is equivalent to

$$
\left\{\begin{array}{l}
x_{j+1}=F\left(x_{j}\right) \\
x_{n}=a_{n}+\xi_{n}
\end{array}\right.
$$

for $a_{n} \in \mathbb{Z}, 0 \leq \xi_{n}<1$.

From these we get the following recursion formula for $a_{i}$ :

$$
a_{0}=0, \quad a_{1}=i_{0}, \quad a_{2}=2 i_{0}+i_{1}, \quad a_{3}=4 i_{0}+2 i_{1}+i_{2}, \ldots
$$

In the case $x=1 / 2$ we obtain by the periodicity of $K\left(f_{a}\right)$ that

$$
\begin{aligned}
a_{k n} & =2^{k n-1} i_{0}+2^{k n-2} i_{1}+\cdots+2^{k(n-1)} i_{n-1}+2^{k(n-1)-1} i_{0}+\cdots+i_{n-1} \\
& =i_{0} 2^{n-1} \frac{2^{k n}-2^{n-1}}{2^{n}-1}+\cdots+i_{n-1} \frac{2^{k n}-1}{2^{n}-1} .
\end{aligned}
$$

Since we know that the limit exists (see [19, Theorem 3.1]), we get

$$
\lim _{j \rightarrow \infty} \frac{F_{a}^{j}(1 / 2)}{2^{j}}=\lim _{k \rightarrow \infty} \frac{a_{k n}}{2^{k n}}=\frac{i_{0} 2^{n-1}+\cdots+i_{n-1}}{2^{n}-1},
$$

which is the statement of our theorem.

4. Aperiodic kneading sequences. In this section we study the monotonicity for $f_{a}$ from the double standard family of maps for which there is an aperiodic kneading sequence. We prove that the itinerary map is strictly increasing in this case. Furthermore, we show how to relate the work in the present paper to the "Real Fatou Conjecture".

TheOREM 4.1. Assume that $K\left(f_{a_{1}}\right)$ and $K\left(f_{a_{2}}\right)$ are aperiodic kneading sequences. Then the two maps are topologically conjugate and both are conjugate to the doubling map of the circle

$$
x \mapsto 2 x(\bmod 1) .
$$

THEOREM 4.2. If $f=f_{a}$ from the double standard family has an aperiodic kneading sequence, then the itinerary map $x \mapsto I(x)$ is strictly increasing, i.e.,

$$
x<y \Rightarrow I(x)<I(y) .
$$

Before we prove Theorem 4.2 we recall the following definition.

DEFINITION 4.3. We say that $J$ is a wandering interval if:

1. $\left\{f^{j}(J)\right\}_{j=0}^{\infty}$ are disjoint.

2. The $\omega$-limit set of $J$ is not a single periodic orbit. 
Let $N$ be either the interval $[-1,1]$ or the circle $\mathbb{T}$. We say that a critical point $c$ of a $C^{2}$ map $f: N \rightarrow N$ is non-flat if there is a $C^{2}$ local diffeomorphism $\varphi$ with $\varphi(c)=0$ such that $f(x)= \pm|\varphi(x)|^{\alpha}+f(c)$ in a neighborhood of $c$.

The double standard family is obviously a circle map with non-flat critical points.

The proof of Theorem 4.2 will follow from the following result obtained in [13]. For an exposition see [15, Chapter 4].

Theorem 4.4 ([15, Chapter 4, Theorem A]). If $f$ is a $C^{2}$ map with non-flat critical points, then $f$ has no wandering intervals.

Proof of Theorem 4.2. Let $x<y$ and $I(x)=I(y)$ and define $J=(x, y)$. We first claim $\left\{f^{j}(J)\right\}_{j=0}^{\infty}$ must be disjoint. Suppose not. Then there are $n \geq 0$ and $k>0$ such that $f(J)$ and $f^{n+k}(J)$ are not disjoint. Thus $K=\bigcup_{p>0} f^{n+k p}(J)$ is an interval. Form $L=\bigcup_{j=0}^{p-1} f^{j}(K)$. We know that $L$ is not the entire circle $\mathbb{T}$ since $I(x)=I(y)$, and $p$ and $q$ cannot be in $\left\{f^{j}(J)\right\}_{j=0}^{\infty}$. Obviously $f(K) \subset K$ and then $\left.f\right|_{L}$ has a sink and hence an attractive periodic orbit. Since the kneading sequence is aperiodic this contradicts Theorem 3.2.

We also have to verify that the $\omega$-limit set of $J$ is not a single periodic orbit. We first claim that the critical point $c=1 / 2$ is in the $\omega$-limit set. Suppose not. Then there is a neighborhood $U$ of the critical point so that for all $x \in J, f^{j}(x) \notin U$ for $j \geq 0$. This means that we can again apply Mañé's theorem. We conclude that there exist constants $C>0$ and $\lambda>1$ so that

$$
\left|D f^{n}(x)\right| \geq C \lambda^{n} \quad \forall n \geq 0 .
$$

This would mean that $\left|f^{n}(J)\right|$ grows exponentially, a contradiction.

Hence $f^{n}(J)$ accumulates on $c$. We have to prove part 2 in the definition of a wandering interval. Suppose that the $\omega$-limit set of $J$ is a periodic orbit. Since $c$ is in the $\omega$-limit set the periodic orbit must contain $c$. Hence $f_{a}$ has a superattractive periodic orbit and $c$ and the kneading sequence is periodic. This is a contradiction. We conclude that $I(x)<I(y)$ and the theorem is proved.

Proof of Theorem 4.1. Suppose $f_{a}$ has an aperiodic kneading sequence. Let $\varphi(x)=I_{f}(x)$ be the itinerary map and $D(x)$ be the doubling map

$$
D(x)=2 x(\bmod 1) .
$$

It is then clear that $\varphi$ is a homeomorphism and $f=\varphi^{-1} \circ D \circ \varphi$, so the conjugacy is proven.

TheOREM 4.5. Assume that $K\left(f_{a_{1}}\right)$ and $K\left(f_{a_{2}}\right)$ are aperiodic kneading sequences such that $K\left(f_{a_{1}}\right)=K\left(f_{a_{2}}\right)$. Then $a_{1}=a_{2}$. 
Proof. Since the map $a \mapsto K(a)$ is non-decreasing, i.e.

$$
a_{1}<a_{2} \Rightarrow K\left(a_{1}\right) \leq K\left(a_{2}\right),
$$

it is enough to prove that there are no intervals where $K(a)$ is constant. But by the density of hyperbolicity [11], in such a parameter interval there must be a point $a^{\prime}$ with an attractive periodic orbit. But for this parameter $a^{\prime}$, by Theorem 3.2, $K\left(a^{\prime}\right)$ is periodic, and this is a contradiction.

It is clear that Theorem 4.5 is really equivalent to the density of parameters with attractive periodic orbits ("The Real Fatou Conjecture") in our present case of the double standard map.

We can realize this as follows. Let $a^{*}$ be a parameter point. We want to prove that $a^{*}$ can be approximated by a sequence of points $a_{n}$ such that $K\left(a_{n}\right)$ is periodic and therefore corresponds to an attractive periodic orbit. If $K\left(a^{*}\right)$ itself is periodic there is nothing to prove. If $K\left(a^{*}\right)$ is aperiodic we can approximate it from below by a sequence $K_{n}$ of increasing periodic kneading sequences. Since $a \mapsto K(a)$ is increasing and continuous, by the intermediate value theorem there is an increasing sequence $\left\{a_{n}\right\}_{n=0}^{\infty}$ so that $K\left(a_{n}\right)=K_{n}$. Obviously $a_{n}$ tends to a limit, which we denote by $a^{\prime}$. By the continuity of $a \mapsto K(a), K\left(a^{\prime}\right)=K\left(a^{*}\right)$. If $a^{\prime}<a$, there would be an interval of parameters with constant aperiodic kneading sequence, which is not possible according to Theorem 4.5.

The following conjecture is in many cases implied by Theorem B of [11].

Conjecture 4.6. Suppose that $K\left(a_{1}\right)=K\left(a_{2}\right)$ are two aperiodic kneading sequences. Then the corresponding maps $f_{a_{1}}$ and $f_{a_{2}}$ are quasisymmetrically conjugate.

An independent proof of Conjecture 4.6, in particular, if it could be done with purely real methods, would be of interest. This would lead to a different proof of Theorem 4.5.

5. Abundance of positive Lyapunov exponents and invariant measures. We first state a theorem which corresponds to a result which in the case of the quadratic family is a consequence of results of M. Misiurewicz from his famous paper [16].

THEOREM 5.1. If $a$ is such that $K(a)$ is preperiodic but not periodic, then $f_{a}$ has an absolutely continuous invariant measure.

We will not give the proof here since it can be given following the ideas given by Misiurewicz. See also the book [7] for an exposition of this proof.

One would expect that the set of parameters satisfying the condition that $K(a)$ is preperiodic but not periodic is of Lebesgue measure 0 . In the case of the quadratic family the corresponding result is due to D. Sands 
[22]. Because of this fact the following result of Collet-Eckmann type is of interest:

TheOREM 5.2. There is a subset $A \subset[0,1]$ of positive Lebesgue measure and constants $c$ and $C>0$ such that for all $a \in A$,

$$
\left|D f_{a}^{n}\left(f_{a}(1 / 2)\right)\right| \geq C e^{c n}, \quad \forall n \geq 0 .
$$

We will not give the proof of this result either since it can be given along the same principles as the proof of the corresponding theorem in the case of the quadratic family following e.g. [4] and [5].

Corollary 5.3. For $a \in A$ the map $f_{a}$ has an absolutely continuous invariant measure.

As a direct consequence of Theorem 5.2 it follows immediately that $D_{n}=$ $\left|D f_{a}^{n}(f(1 / 2))\right|$ satisfies

$$
\sum_{n=0}^{\infty} D_{n}^{-1 / l}<\infty .
$$

In our case $l=3$, since this is the power-law behavior of the maps at the critical inflexion point $1 / 2$.

This indicates that an analogy to a result of Nowicki-van Strien, [21, Main Theorem], proved in the interval case, should be applicable, and if so we conclude that there is an absolutely continuous invariant measure.

It is also clear that a direct proof of Corollary 5.3 can also be given following the ideas of e.g. [4, part II], or [6].

6. Conjectures and further results. It seems reasonable that the analogy to the quadratic family could be carried even further. In particular, Nowicki, Martens and Lyubich [14] proved that the parameter space of the quadratic family $x \mapsto q_{a}(x)=1-a x^{2}, 0<a<2$, can be written as a union

$$
(0,2)=A \cup B \cup S \quad \text { a.e., }
$$

where $A$ is the set of parameters for which $q_{a}$ has an absolutely continuous invariant measure, $B$ is the set of parameters for which $q_{a}$ has an attractive periodic orbit, and $S$ is the set of parameters for which $q_{a}$ has a singular non-atomic invariant measure $\mu_{s}$, which is also the unique physical measure, i.e. a.e. point in the dynamic interval is Birkhoff generic:

$$
\sum_{k=0}^{n-1} \delta_{f^{k}(x)} \rightarrow \mu_{s} \quad \text { for a.e. } x,
$$

in the weak-* topology.

Later Lyubich [12] proved that $S$ is of Lebesgue measure 0. It seems natural to conjecture that the corresponding result is true for the double standard family for $b=1$. 
Conjecture 6.1. For the double standard family $f_{a}, 0 \leq a<1$ the parameter space can be subdivided into three sets

$$
[0,1]=A \cup B \cup S \quad \text { a.e. }
$$

so that

(i) A, the set of a's such that $f_{a}$ has an absolutely continuous invariant measure, is of positive Lebesgue measure;

(ii) $B$, the set of a's such that $f_{a}$ has a stable periodic orbit, is open and dense;

(iii) $S$, the set of parameters for which $f_{a}$ has a unique non-atomic singular physical measure, is of Lebesgue measure 0.

Let us finish with some remarks about what is known about this conjecture.

(i) is Theorem 5.2 above. (ii) is the Real Fatou Conjecture in this case and is a result of Levin and van Strien [11]. The subdivision (6.1) and (iii) are open problems in the case of the double standard family even if these facts are both known for the quadratic family.

Acknowledgements. The second author was supported by FCT Grant BPD $/ 36072 / 2007$. The research of AR supported in part by Centro de Matemática da Universidade do Porto (CMUP) financed by FCT through the programmes POCTI and POSI, with Portuguese and European Community structural funds. The second author would like to thank for the hospitality at the departments of mathematics of KTH and UAB where this work was done. The authors also want to thank the Göran Gustafsson Foundation $\mathrm{UU} / \mathrm{KTH}$ for financial support.

\section{References}

[1] L. Alsedà and F. Mañosas, The monotonicity of the entropy for a family of degree one circle maps, Trans. Amer. Math. Soc. 334 (1992), 651-684.

[2] V. I. Arnold, Remarks on perturbation theory for problems of Mathieu type, Uspekhi Mat. Nauk 38 (1983), no. 4, 189-203 (in Russian).

[3] - Small denominators I. Mappings of the circumference onto itself, Amer. Math. Soc. Transl. 46 (1965), 213-284.

[4] M. Benedicks and L. Carleson, On iterations of $1-a x^{2}$ on $(-1,1)$, Ann. of Math. (2) 122 (1985), 1-25.

[5] - - - The dynamics of the Hénon map, ibid. (2) 133 (1991), 73-169.

[6] M. Benedicks and L.-S. Young, Absolutely continuous invariant measures and random perturbations for certain one-dimensional maps, Ergodic Theory Dynam. Systems 12 (1992), 13-37.

[7] P. Collet and J.-P. Eckmann, Iterated Maps on the Interval as Dynamical Systems, Progr. Phys. 1, Birkhäuser, Boston, MA, 1980. 
[8] J. Graczyk and G. Świątek, The Real Fatou Conjecture, Ann. of Math. Stud. 144, Princeton Univ. Press, Princeton, NJ, 1998.

[9] J. Guckenheimer, Sensitive dependence to initial conditions for one dimensional maps, Comm. Math. Phys. 70 (1979), 133-160.

[10] K. Hockett and P. Holmes, Bifurcation to badly ordered orbits in one-parameter families of circle maps or angels fallen from the devil's staircase, Proc. Amer. Math. Soc. 102 (1988), 37-65.

[11] G. Levin and S. van Strien, Bounds for maps of an interval with one critical point of inflection type. II, Invent. Math. 141 (2000), 399-465.

[12] M. Lyubich, Almost every real quadratic map is either regular or stochastic, Ann. of Math. (2) 156 (2002), 1-78.

[13] M. Martens, W. de Melo and S. van Strien, Julia-Fatou-Sullivan theory for real one-dimensional dynamics, Acta Math. 168 (1992), 273-318.

[14] M. Martens and T. Nowicki, Invariant measures for typical quadratic maps, in: Géométrie complexe et systèmes dynamiques (Orsay, 1995), Astérisque 261 (2000), 239-252.

[15] W. de Melo and S. van Strien, One-Dimensional Dynamics, Ergeb. Math. Grenzgeb. 25, Springer, Berlin, 1993.

[16] M. Misiurewicz, Absolutely continuous measures for certain maps of an interval, Inst. Hautes Études Sci. Publ. Math. 53 (1981), 17-51.

[17] M. Misiurewicz and A. Rodrigues, Non-generic cusps, Trans. Amer. Math. Soc., to appear.

[18] -, - On the tip of the tongue, J. Fixed Point Theory Appl. 3 (2008), 131-141.

[19] —, -, Double standard maps, Comm. Math. Phys. 273 (2007), 37-65.

[20] M. Misiurewicz and E. Visinescu, Kneading sequences of skew tent maps, Ann. Inst. H. Poincaré Probab. Statist. 27 (1991), 125-140.

[21] T. Nowicki and S. van Strien, Invariant measures exist under a summability condition for unimodal maps, Invent. Math. 105 (1991), 123-136.

[22] D. Sands, Misiurewicz maps are rare, Comm. Math. Phys. 197 (1998), 109-129.

[23] J.-C. Yoccoz, manuscript.

Michael Benedicks

Matematiska Institutionen

$\mathrm{KTH}$

S-100 44 Stockholm, Sweden

E-mail: michaelb@math.kth.se
Ana Rodrigues

Department of Mathematical Sciences

IUPUI

402 N. Blackford Street, Indianapolis IN 46202-3216, U.S.A.

and

CMUP, Rua do Campo Alegre, 687

4169-007 Porto, Portugal

E-mail: arodrig@math.iupui.edu

Received 16 January 2009;

in revised form 2 July 2009 